\title{
The Mu2e tracker and calorimeter systems
}

\author{
Simona Giovannella*† \\ Laboratori Nazionali di Frascati dell'INFN \\ Via Enrico Fermi 40, 00044, Frascati (Roma), Italy \\ E-mail: simona.giovannellaelnf.infn.it
}

The Mu2e experiment at Fermilab aims to observe the charged-lepton flavor violating neutrinoless conversion of a negative muon into an electron. The conversion results in a monochromatic electron with an energy slightly below the muon rest mass (104.97 MeV). Two major elements of the detector system are the straw tube tracker and the crystal calorimeter. This paper describes the tracker and the calorimeter systems and provides experimental results obtained in the prototyping and pre-production phases.

The European Physical Society Conference on High Energy Physics

5-12 July, 2017

Venice

* Speaker.

$\dagger$ on behalf of the Mu2e Collaboration. 


\section{The Mu2e Experiment}

The Mu2e experiment [1] at Fermilab will measure the charged-lepton flavor violating (CLFV) neutrino-less conversion of a negative muon into an electron in the field of a nucleus. This process results in a mono-energetic electron with an energy slightly below the muon rest mass (104.967 $\mathrm{MeV})$. Mu2e will improve the previous measurement by four orders of magnitude using a new technique, reaching a single event sensitivity (SES) of $2.5 \times 10^{17}$ on the conversion rate. The experiment will reach mass scales of nearly $10^{4} \mathrm{TeV}$, far beyond the direct reach of colliders. The experiment is sensitivity to a wide range of new physics, complementing and extending other CLFV searches.

A very intense pulsed muon beam $\left(\sim 10^{10} \mu / \mathrm{s}\right)$ is stopped on an aluminium target inside a very long solenoid where the detector is located (Fig. 1). The Mu2e tracker is the primary device to measure the momentum of the electron and separate it from background. The crystal calorimeter plays a crucial role in providing particle identification capabilities and a fast online trigger filter, while aiding the track reconstruction capabilities. An external veto for cosmic rays surrounds the solenoid. An extinction monitor detects scattered protons from the production target to evaluate the fraction of out-of-time beam and a stopping target monitor measures the rate and the number of negative muons that stop in the target.

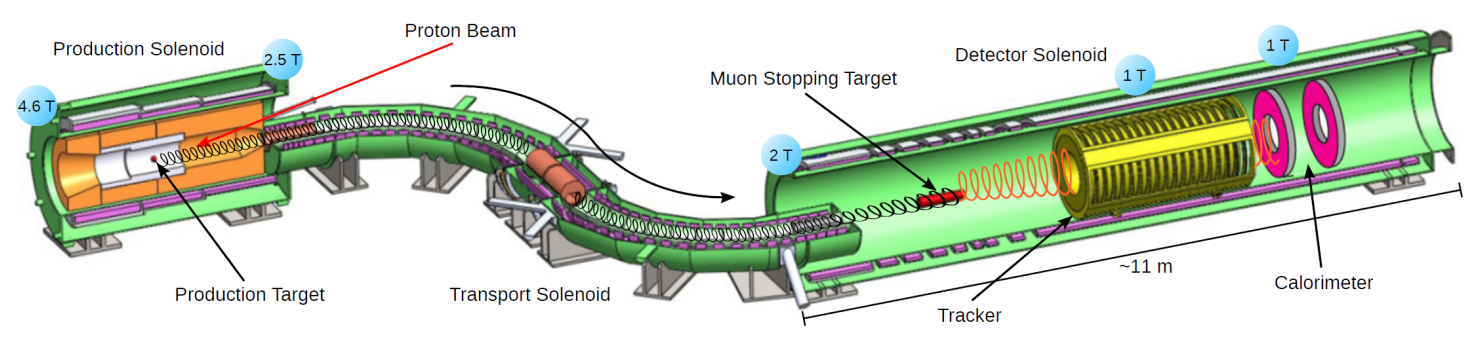

Figure 1: The Mu2e detector. Cosmic Ray Veto, Extinction Monitor and Stopping Target Monitor are not shown.

In the following, details of the tracker and of the calorimeter will be discussed, providing a summary of the experimental status for the two detectors.

\section{Tracker}

The Mu2e tracker system [2] has been designed to maximizing acceptance for conversion electrons (CE), minimizing the contamination from the muon Decay-In-Orbit (DIO) background. Nuclear modifications push the DIO spectrum towards the CE signal; energy loss and detector resolution produce overlap of the two processes. The selected design is based on nearly 20,000 low mass straw drift tubes of $5 \mathrm{~mm}$ in diameter, with $15 \mu \mathrm{m}$ Mylar wall and $25 \mu \mathrm{m}$ sense wire. Straws are oriented transversally to the solenoid axis and arranged in 18 stations for a total lenght of 3.2 metres. A central hole, $38 \mathrm{~cm}$ in diameter, makes the device blind to low momentum background particles.

Tracker performance has been studied by Monte Carlo using Mu2e full simulation. The core momentum resolution of $115 \mathrm{keV} / \mathrm{c}$, with a $3 \%$ high tail slope of $179 \mathrm{keV} / \mathrm{c}$, is well within physics 


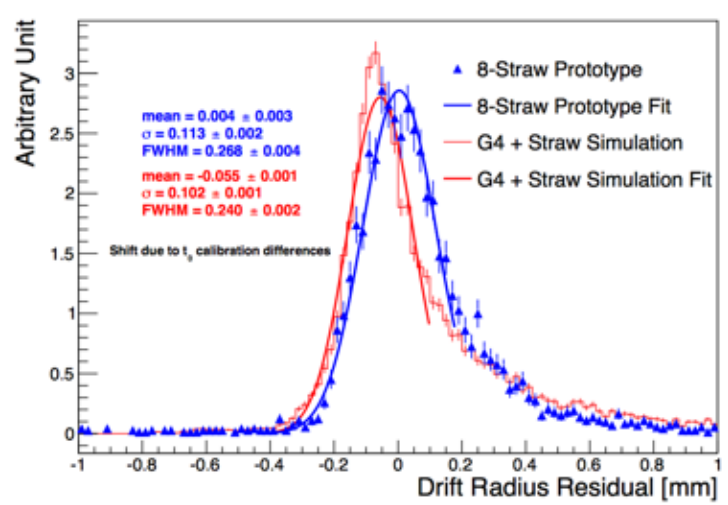

Transverse Resolution
(Data vs MC)
$\sigma_{\text {data }}=0.113 \pm 0.002 \mathrm{~mm}$
$\sigma_{M C}=0.102 \pm 0.001 \mathrm{~mm}$

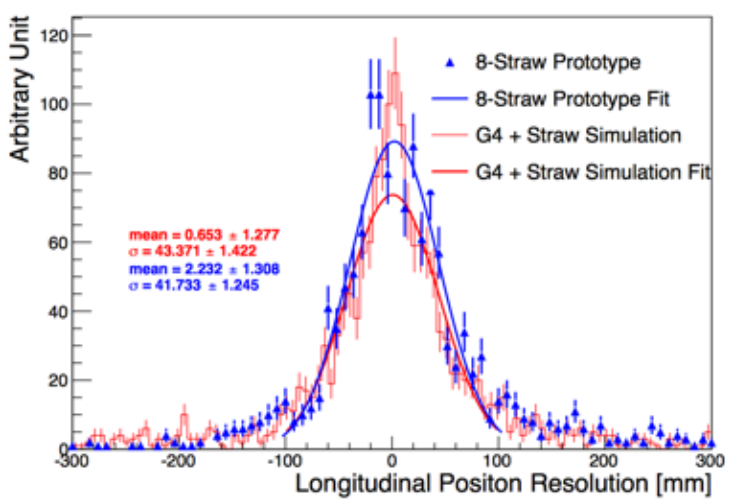

Longitudinal Resolution

(Data vs MC)

$\sigma_{\text {data }}=42 \pm 1 \mathrm{~mm}$

$\sigma_{M C}=43 \pm 1 \mathrm{~mm}$

Figure 2: Transverse (left) and longitudinal (right) position resolution for an eight channel prototype of the tracker. Data from minimum ionizing particles (blue triangles) are compared with Monte Carlo simulation (red crosses). Resolution is extracted with Gaussian fits to the spectra.

requirements and stable when incresing accidental hit rate. The total track efficiency of $\sim 9 \%$ is fully dominated by geometric acceptance.

An eight channel tracker prototype has been built and tested with cosmics. In Fig. 2 the extracted position resolution is compared with Monte Carlo expectations. The shift observed in the transverse resolution is due to the $T_{0}$ calibration differences. A first pre-production prototype with final design was recently built and is being tested. A vertical slice test on fully instrumented panels with the entire FEE chain will follow.

\section{Calorimeter}

The Mu2e calorimeter [3] has to provide confirmation for CE signal events, a powerful $e / \mu$ separation - with a muon rejection factor of $\sim 200$, a standalone trigger and seeding for track reconstruction. An energy resolution of $O(5 \%)$ and a time resolution $<500$ ps for $100 \mathrm{MeV}$ electrons are sufficient to fulfill these requirements. The calorimeter design consists of two disks made by 674 undoped CsI scintillating crystals with $(34 \times 34 \times 200) \mathrm{mm}^{3}$ dimension. Each crystal is read-out by two custom array large area $\left(2 \times 3\right.$ of $6 \times 6 \mathrm{~mm}^{2}$ cells $)$ UV-extended Silicon PhotoMultipliers (SiPMs). The crystals will receive an ionizing dose of $90 \mathrm{krad}$ and a fluence of $3 \times 10^{12}$ $\mathrm{n} / \mathrm{cm}^{2}$ in three years running. The photosensors, being shielded by the crystals, will get a three times smaller dose.

A small calorimeter protoype, a $3 \times 3$ array of CsI crystals coupled to a single multi-pixel photon counter, has been tested at the Frascati Beam Test Facility with electron beams of 80-120 $\mathrm{MeV}$ [4]. A time resolution of $100 \mathrm{ps}$ and an energy resolution of $6.5 \%$ has been obtained for 100 $\mathrm{MeV}$ particles. For the latter, a significant leakage contribution is present, confirmed by simulation. 

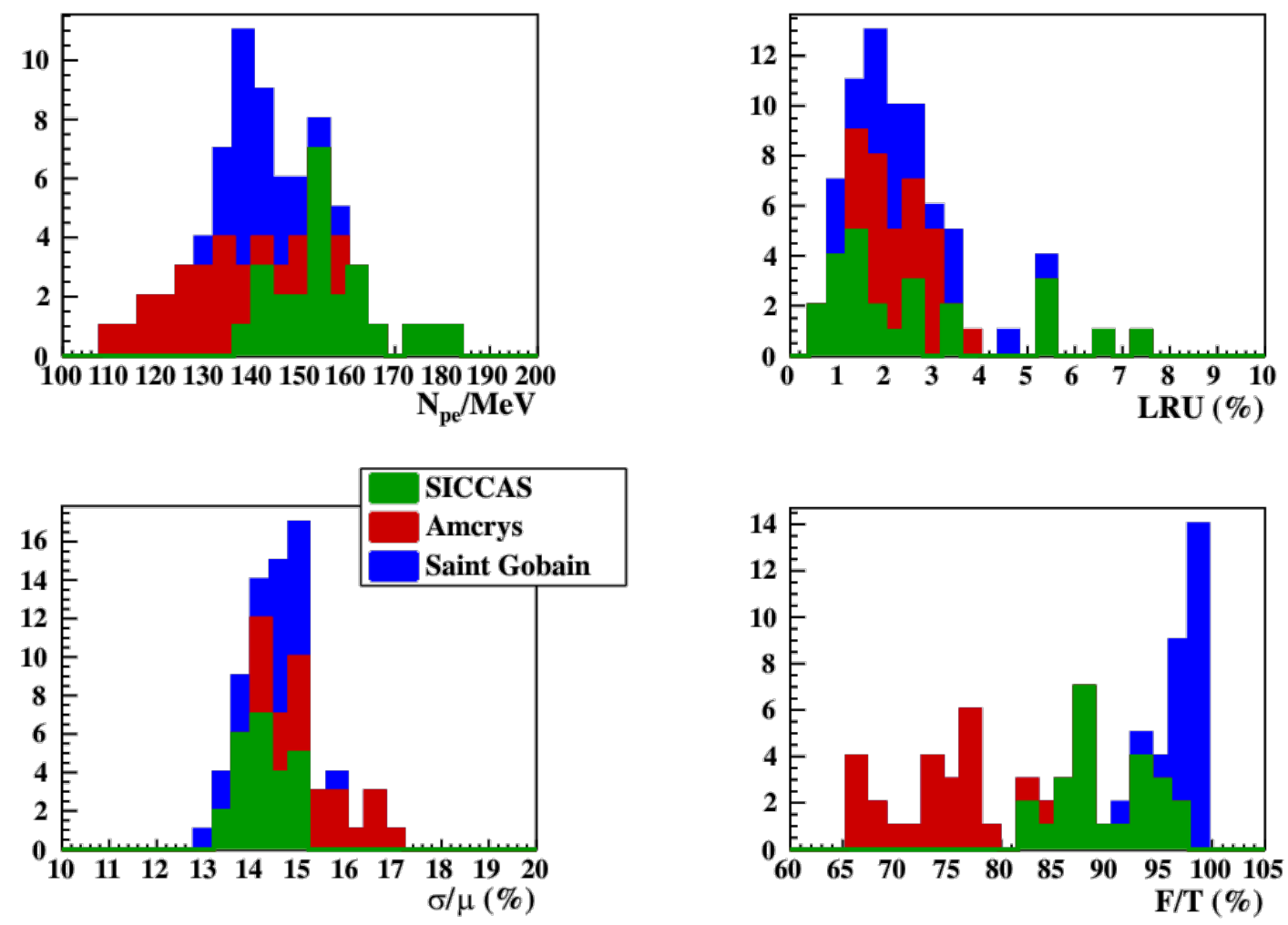

Figure 3: Summary of the optical properties for pre-production CsI crystals, coupled to UV-extended photomultiplier and tested with $511 \mathrm{keV}$ photons: light yield (top left), longitudinal repsonse uniformity (top right), energy resolution (bottom left) and fraction of the fast component (bottom right).

Pre-production components both for crystals and SiPMs have been received from different vendors. They have been characterized and irradiation test have been carried out for a small subsamples. Some results are shown in Figs. 3, 4. Pre-production components have been used to build a large calorimeter prototype, with 51 crystals ans 102 SiPMs and front end boards, testing integration and assembly procedures.

\section{Conclusions}

The Mu2e experiment will exploit the world's highest intensity muon beams of the Fermilab Muon Campus to search for CLFV, improving current sensitivity by a factor $10^{4}$. A low mass straw tube tracker and a pure CsI crystal calorimeter with SiPM readout have been selected to satisfy the demanding requirements. Both systems are concluding the prototyping phase. Production of final components is starting, and it will move to full regime by the end of 2017. Detector installation is foreseen in 2020, followed by Mu2e commissioning and data taking.

\section{Acknowledgments}

We are grateful for the vital contributions of the Fermilab staff and the technical staff of the 

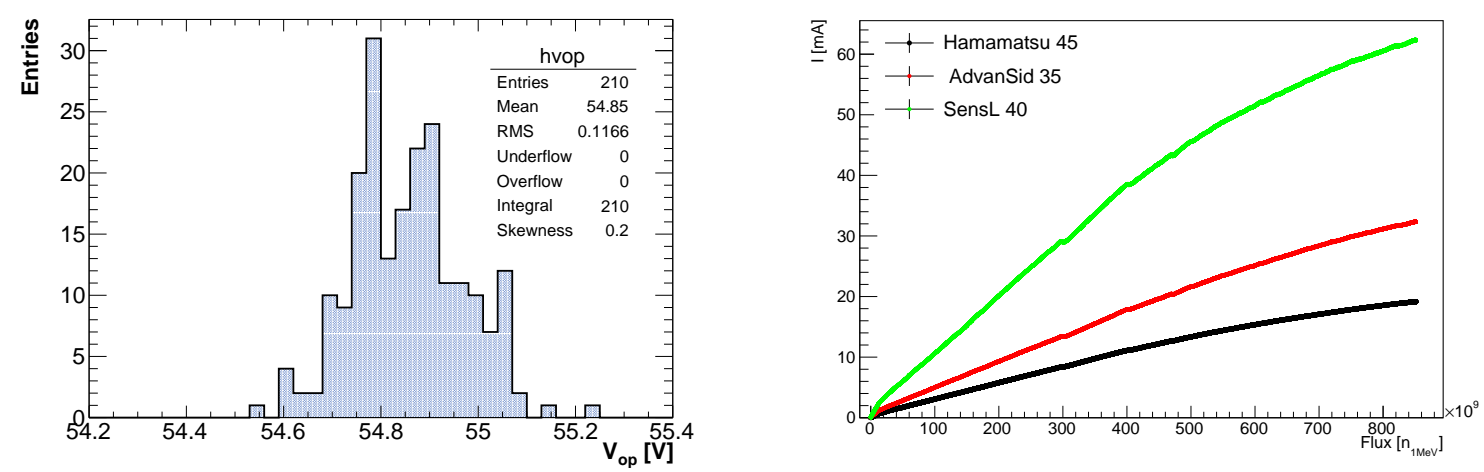

Figure 4: Left: operating voltage of pre-production SiPMs from Hamamatsu. Right: dark current as a function of the neutron flux for pre-production SiPMS from Hamamatsu (black), AdvanSiD (red) and SensL (green).

participating institutions. This work was supported by the US Department of Energy; the Italian Istituto Nazionale di Fisica Nucleare; the Science and Technology Facilities Council, UK; the Ministry of Education and Science of the Russian Federation; the US National Science Foundation; the Thousand Talents Plan of China; the Helmholtz Association of Germany; and the EU Horizon 2020 Research and Innovation Program under the Marie Sklodowska-Curie Grant Agreement No.690385. Fermilab is operated by Fermi Research Alliance, LLC under Contract No. De-AC02-07CH11359 with the US Department of Energy, Office of Science, Office of High Energy Physics. The United States Government retains and the publisher, by accepting the article for publication, acknowledges that the United States Government retains a non-exclusive, paid-up, irrevocable, world-wide license to publish or reproduce the published form of this manuscript, or allow others to do so, for United States Government purposes.

\section{References}

[1] L. Bartoszek et al., "Mu2e Technical Design Report”, ArXiv:1501.05241, Fermilab-TM-2594, Fermilab-Design-2014-01.

[2] M. J. Lee on behalf of the Mu2e collaboration, "The Straw-tube Tracker for the Mu2e Experiment", Nuclear and Particle Physics Proceedings 273-275 (2016) 2530.

[3] N.Atanov et al., "Design and status of the Mu2e electromagnetic calorimeter", Nucl. Instr. and Meth. A 824 (2016) 695.

[4] O. Atanova et al., "Measurement of the energy and time resolution of a undoped CsI + MPPC array for the Mu2e experiment", JINST 12 (2017) P05007, ArXiv:1702.03720. 